\title{
Grid Models versus TIN: Geometric Accuracy of Multibeam Data Processing
}

Alain DE WULF ${ }^{1}$, Denis CONSTALES ${ }^{2,3}$, Timothy NUTTENS $^{1}$, Cornelis STAL $^{1}$

${ }^{1}$ Ghent University, Department of Geography

${ }^{2}$ Ghent University, Department of Mathematical Analysis

${ }^{3}$ Ghent University, Laboratory for Chemical Technology

Topic C: innovations in processing techniques

\section{INTRODUCTION}

Multibeam echosounder measurements serve to make a digital terrain model of the sea floor. The Delaunay triangulation is a widely appreciated and investigated mathematical model to represent the sea floor topography and is highly efficient for building triangular irregular networks (TINs) out of non-homogeneous data such as raw multibeam data.

Obtaining an accurate model of the sea floor is a major concern in dredging works. Contemporary hydrographical surveying tools, especially the multibeam echosounder, yield a very dense sampling of the sea floor. Consequently, this immense amount of data needs to be processed to generate an accurate terrain model, according to time and accuracy constraints imposed by the client. Modeling can be carried out in post-processing or in real-time, performing a real-time accountability which keeps track of the "cut" or "fill" volume changes realized at that moment. Most multibeam systems deliver equidistant interpolated data, allowing faster processing to be achieved by using equidistant grid modeling. Both modeling techniques (TIN and grid) yield their own advantages and drawbacks. In this paper, the standard deviation of the volume computed directly in a TIN model or indirectly by interpolating a (usually equidistant) point set in a TIN model is derived.

\section{SEA FLOOR MAPPING}

Obtaining an accurate model of the sea floor is a major concern in dredging works. Nowadays' hydrographic surveying tools, especially the multibeam echosounder, yield a very dense point sampling of the sea floor. The immense amount of data needs to be processed (e.g. filtering or data reduction), to form an accurate terrain model, according to some constraints imposed by the client. Modeling can be performed in post-processing or in real-time. Performing a real-time accountability will keep track of the haul, realized at some moment.

DTM (Digital Terrain Model) software for hydrographic purposes must meet the following four requirements:

1. Fast model creation: the purpose is to create the model as fast as the data is gathered, so that real-time control and verification are possible;

2. Editing (manual or automatic) of the model: adding data points as well as deleting data points (vertices) in the model are both required. When examining the theoretical model of a site, intervening directly in the model as it is displayed on the computer monitor is a prerequisite, by relocating, deleting or adding vertices. It should also be possible to replace data from resurveyed areas with more recent data, and to update the existing model with this new information;

3. To include the possibility of data reduction: reduce the large amounts of multibeam data to acceptable levels, keeping the sea floor model as accurate as possible, but the data set manageable for the used computers;

4. Data quality control: the final result in the form of volume calculations should be as close to the truth as possible and certainly not further away than acceptable, assuming that the acceptable quality level is realizable. In order to qualify the data, different statistical tools can be applied (Höhle \& Höhle, 2009). The final data model and the derived volume computation should give a correct approximization of the real situation. The acceptability of the model is related to the measurement device, as well as to the requirements of the client.

Grid models and TINs are the most frequently used models in hydrography (Brouns, De Wulf and Constales 2001), offering different kinds of advantages and drawbacks. Both terrain representations are discussed with their advantages and drawbacks. Grid based filtering approaches (Wack and Wimmer 2002) and TIN based filtering approaches are intensively discussed in the field of airborne laser scanning, using similar techniques. The distinction between TIN based and grid based filtering 
techniques can be made by respectively an original point set filtering and a filtering based on interpolated equidistant cells.

In general, filtering techniques can be divided in four categories (Krzystek 2003);

- $\quad$ Least squares interpolation(Briese and Kraus 2003);

- $\quad$ Local slope based filtering (Vosselman 2000; Sithole 2001);

- $\quad$ Morphological filters (Zhang et al. 2003);

- $\quad$ Convex hull filtering (Krzystek 2003).

\section{GRID MODELING}

Nowadays, most multibeam systems offer equidistant grid data as default output of the on-line and on-board processing chain. The plane coordinate system used is generally a square grid with the axis parallel to the Easting and Northing axis of the grid coordinate system used. Since the use of GNSS (Global Navigation Satellite System) equipment, the universal transverse Mercator coordinate system (UTM) in relation to the ETRS89 datum (referencing the global ellipsoid GRS80) has established itself as the standard grid system in Europe.

Height/depth values can be related to a universal reference (e.g. the WGS84 and GRS80 ellipsoid, LAT, geoid...) or, in most cases, to a conventional reference plane. In the latter case, if the GNSS receiver onboard the vessel gives the height above the WGS84 ellipsoid, a conversion matrix between ellipsoid and the reference plane used should be given. Alternatively, the older technique of tide gauges can be used, in which case the measured depths are related to the water surface and the water surface is related to the reference plane by means of tide gauges. This leaves the grid interval distance as the unique and most important user-defined parameter.

The use of equidistant points allows to store only the depth values in computer memory and not the Easting and Northing values, since these values can be computed out of the row and column number of each point, assuming (for instance) that the point storage is performed in a row-wise manner in the computer memory. Memory use can be optimized by using arrays of integer values that, for dredging based surveys, can be limited to only 2 byte for each depth or point, giving a range of 216 or 65536 height/depth values, or a range of approximately $65 \mathrm{~m}$ with the $\mathrm{mm}$ as unit.

\section{TIN BASED MODELING}

\section{Principle}

It is a common practice to use the Delaunay triangulation (Brouns, De Wulf, and Constales 2003) to construct a TIN rather than other, less restrictive, triangulations. In a Delaunay triangulation, the circumscribing circle of any triangle contains no other vertices (Shewchuk 1996).

Triangles whose circumcircle does contain another vertex are invalid and need to be replaced by another triangle by a process called edge flipping; this principle is shown in Figure 1 (left, middle). The triangles $a b c$ and $a c d$ are not Delaunay triangles, since they contain respectively $d$ and $b$ in their circumscribing circles. After flipping the edge $a c$ to $b d$, the triangles $a b d$ and $b c d$ are created, which do not contain other vertices in their circumscribing circle. They therefore meet the Delaunay requirement.

Figure 1 (right) represents what is called edge completion: when four points are co-circular, the resulting quadrilateral is (arbitrarily) split in two separate triangles. This constitutes a degenerated case as either of the two diagonals can be constructed. 

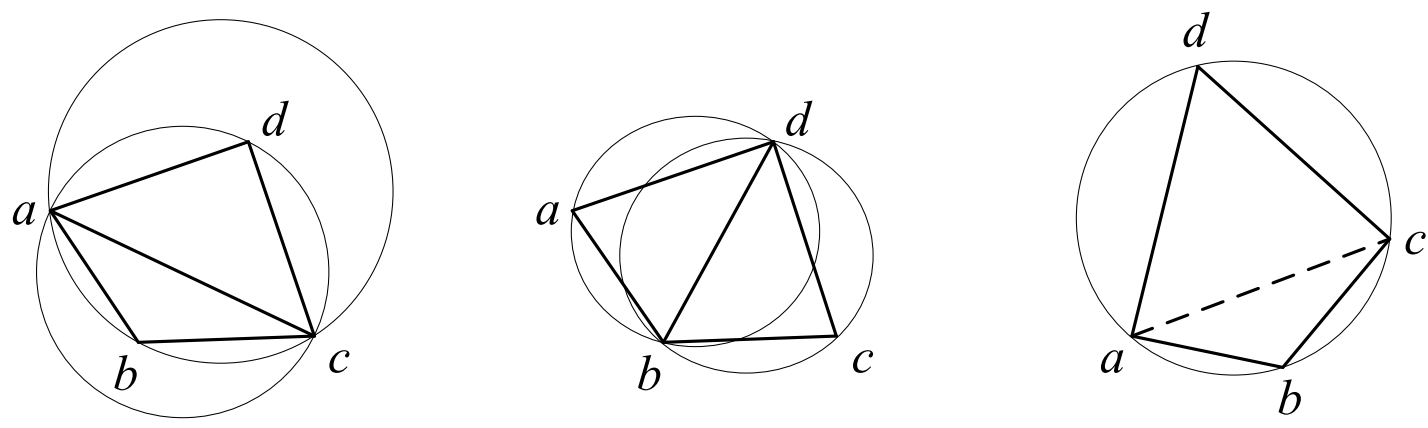

Figure 1: Delauney triangle principle

It can be proven (Shewchuk 1996) that the Delaunay triangulation of a set of vertices is unique; this is an important quality asset towards the client, as it allows him to repeat the calculations to verify the results independently.

\section{Advantages and drawbacks of grid models versus TINs}

It can be a requirement of the client of dredging/reclamation projects that the original measured points have to be in the digital terrain model from which the volumes are derived. This requirement allows the client to check the original results in a 3D modeling environment, which simplifies the validation of the point set. TINs are a favorite scheme to construct a DTM from a sea floor, measured at discrete spots. Grid models, compared to TINs, have three important drawbacks:.

1. It is generally impossible to have each sampled point of a TIN associated to one grid height/depth, since the measurements are not on a regular grid. Instead, the mutual distances depend on the survey ship's survey system (equally spaced measurements or not) and attitude (roll, pitch, yaw);

2. Grid values do not reflect the actual measurements, since gridding means either assigning interpolated values when the measurement density is inferior to the grid density, or resampling and loss of information, when the measurement density is superior to the grid size, the latter introduces unwanted "smoothing" in the DTM;

3. The grid model is not adaptive: whereas TINs will naturally represent areas with detailed relief information with a denser triangle pattern than areas with a smoother relief, grids will be far less flexible to cope with variable levels of detail.

TINs do not have these drawbacks, but they are more demanding towards computer memory and processing time. Moreover, the algorithms needed for geometric computations are more sophisticated.

\section{ACCURACY OF TIN BASED VOLUMES}

\section{Volume computation in a TIN}

Volume computations in TINs or grid models are quite straightforward. In a TIN model, a prismatic volume is computed between a horizontal reference level and each triangle, on the condition that in planimetry, the triangle is inside the area where the volume has to be computed. Furthermore, it is assumed that for hydrographic volume computations the most important stochastic error is the altimetric error and that the planimetric positioning errors can be neglected as the sea floor is rather flat. Quite often, especially when a difference of volumes has to be computed, an equidistant set of points is interpolated in the TIN(s): this allows to construct a difference model for which the height/depth of each point is the difference of interpolated heights/depths in both TIN models. Hereunder, a synthesis of the mathematical analysis of both cases is performed. We assume a set of $n$ original points that will be triangulated and, in a second step, a set of $m$ points interpolated in the TIN:

$$
\begin{gathered}
\text { TIN }=\left\{f_{i} \in A: A \subset \mathbb{R}^{3}: i=0 \ldots n\right\} \\
\text { Subset }=\left\{\widetilde{f}_{\iota} \in B: B \subset \mathbb{R}^{3}: i=0 \ldots m\right\}
\end{gathered}
$$


The variance of the volume for both steps will be computed, based on a constant variance for each initial height/depth value $f_{i}$. We assume a stochastic error, implying that all systematic errors have already been corrected. With $A_{j}$ the planimetric surface of a triangle $j, f_{\text {ref }}$ the height of the horizontal reference plane and $f_{i}$ the elevation of the three vertices $i$ of the triangle, the volume $V_{j}$ generated by one triangle $j$ is equal to (Figure 2):

$$
\left(\frac{1}{3}\left(f_{1}+f_{2}+f_{3}\right)-f_{r e f}\right) A_{i}=V_{j}
$$

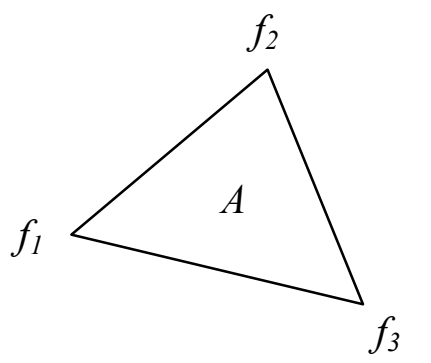

Figure 2: Triangle with elevations $f_{i}$ and surface $\mathrm{A}$

The total volume $V$ of the TIN is the sum of the volumes of all individual prisms, thus:

$$
\frac{1}{3} \sum_{i} f_{i}\left(\sum_{f_{i} \in A_{j}} A_{j}\right)-f_{\text {ref }} A_{\text {total }}=V
$$

If we call $B_{i}$ the sum of the surfaces of all triangles with point $i$ as vertex or:

$$
B_{i}=\left(\sum_{f_{i} \in A_{j}} A_{j}\right)
$$

Then we can write:

$$
\frac{1}{3} \sum_{i} f_{i} B_{i}-f_{\text {ref }} A_{\text {total }}=V
$$

Assuming that all $f_{i}$ are independent, the variance of the volume can be written, as we proved in De Wulf et al. (2012), in either of two equivalent ways:

or:

$$
\operatorname{Var}(V)=\frac{\operatorname{Var}(f)}{9} \sum_{i} B_{i}^{2}
$$

$$
\operatorname{Var}(V)=\operatorname{Var}(f) \frac{1}{n} A_{\text {total }}^{2} \cdot\left[1+\left(\frac{\sigma(B)}{\bar{B}}\right)^{2}\right]
$$


The question we have to solve here is "what is the variance (or loss of accuracy) when we base the volume computation on a set of $m$ random points interpolated in a TIN (Figure 3)?"

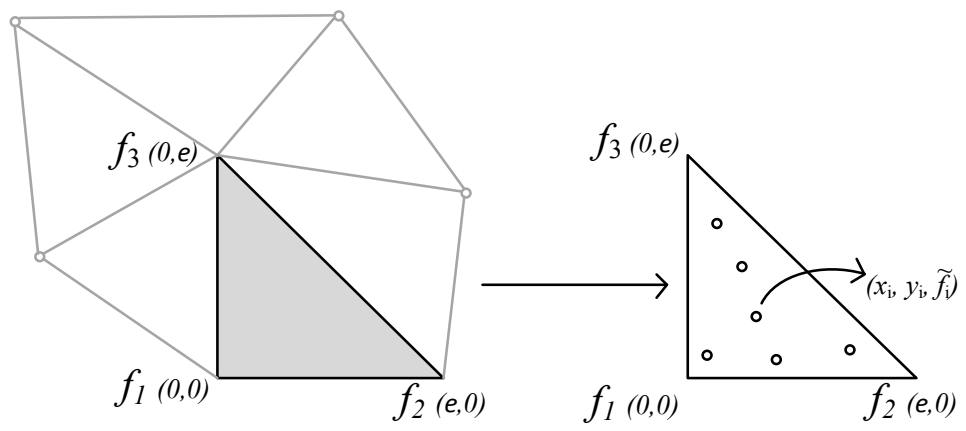

Figure 3: Assumed structure of a triangle with $m_{\Delta}$ points, having elevation values $\tilde{f}_{i}$

Without loss of generality, we can assume a rectangular triangle with corner points at the origin $(0,0)$, the point $(\varepsilon, 0)$ and the point $(0, \varepsilon)$. The result for non-rectangular triangles will prove identical. If the set of $m$ points are equidistant points instead of random points, the variance will be smaller, and the random case under study can be seen as a "worst case" scenario.

We assume that the true height over the triangle can be obtained by linear interpolation, but that the measurement is affected by an error term $\sigma(x, y)$ which is zero on average and whose square's expectation value is the general measurement error. The complete mathematical derivation will be published soon but leads to the result that

$$
\operatorname{Var}(V)=\sum_{\Delta} \frac{A_{\Delta}^{2}}{m_{\Delta}}\left(\frac{1}{4} \operatorname{Var}\left(f_{\Delta}\right)+\operatorname{Var}(f)\right)
$$

The variance is a sum over all triangles, where, within each triangle, $m_{a}$ the number of interpolated points, $\operatorname{Var}(f)$ the a priori assumed stochastic measurement error, $A_{\text {, the }}$ planimetric surface and $\operatorname{Var}\left(f_{3}\right)$ the variance of the heights of the 3 vertices.

Combining both steps, the construction of a TIN and computing the volume based on a new set of $m$ points interpolated in that TIN, the global variance becomes:

$$
\operatorname{Var}(V)=\sqrt{\operatorname{Var}(f) \frac{1}{n} A_{\text {total }}^{2} \cdot\left[1+\left(\frac{\sigma(B)}{\bar{B}}\right)^{2}\right]+\sum_{\Delta} \frac{A_{\Delta}^{2}}{m_{\Delta}}\left(\frac{1}{4} \operatorname{Var}\left(f_{\Delta}\right)+\operatorname{Var}(f)\right)}
$$

\section{CONCLUSIONS}

Multibeam echosounder data impose some specific requirements to the processing. These requirements have been identified and the different aspects of DTM construction by grid modeling and by Delaunay triangulation have been treated in this context and opposed to each other as two alternatives, of which the advantages and drawbacks have been discussed.

Editing the model is significantly more complex when TINs are used in comparison to regular spaced grids. The authors use an adapted merge-step in the divide-and-conquer algorithm to replace old data in an existing triangulation by newly available data. TINs are to be preferred when the surveyed area has a non-homogeneous coverage.

Equidistant grid models are less flexible, but offer higher speed, lower memory requirements and easier implementation algorithms as most important assets, making them to be preferred when the measured area is homogeneously covered by a high-density multibeam survey. For heterogeneous covered areas, typical for singlebeam surveys, TINs are a priori the preferred option. A mathematical form for the standard deviation of TIN based volume computations has been proposed, either based on irregular spaced points or not. 
Prof. Dr. ir. Alain De Wulf is professor, working on quality aspects of geodesy and land surveying in general. He also has a special interest in hydrography and is developing specialized software for the processing and quality assessment of hydrographic 3D acquisition sensors.

Dr. Denis Constales is a researcher both in the Department of Mathematical Analysis and in the Laboratory for Chemical Technology at Ghent University.

MSc. Timothy Nuttens is a teaching assistant at the department of Geography and PhD student working on the use of high accuracy terrestrial laser scanning for deformation measurements of newly built concrete tunnels ('Diabolo Project', Zaventem and 'Liefkenshoek Railway Connection Project', Antwerp, Belgium).

MSc. Cornelis Stal is a PhD student working on the combination of airborne and terrestrial laser scanning for 3D city modelling. His special interest is in the (automatic) generation of geometric, radiometric and semantic rich 3D models, derived from irregular point sets and other spatial datasets.

\section{CONTACT DETAILS}

Prof. dr. ir. Alain De Wulf

Ghent University - Department of Geography

Krijgslaan 281 (Building S8)

B-9000 Ghent, BELGIUM

Tel. +329 2644983

Fax. +3292644985

Email: Alain.DeWulf@UGent.be

Web site: http://geoweb.ugent.be/data-acquisition-3d

\section{REFERENCES}

Briese, C., and K. Kraus. 2003. "Laser-Geländemodelle: Zusammenspiel von Filterung und Datenreduktion". In Photogrammetrie-Fernerkundung-Geoinformation: Auf dem Weg zu operationellen Prozessketten in Photogrammetrie, Fernerkundung und Geoinformation Bochum, Germany: Publikationen der Deutschen Ges.ellschaft für Photogrammetrie, Fernerkundung und Geoinformation. 9 p. (on CD-ROM).

Brouns, G., A. De Wulf, and D. Constales. 2003. "Delaunay triangulation algorithms useful for multibeam echosounding." Journal of Surveying Engineering no. 129 (2):79-84.

Brouns, Gert, Alain De Wulf, and Denis Constales. 2001. "Multibeam data processing: adding and deleting vertices in a Delaunay triangulation." Hydrographic Journal no. 101:3-9.

De Wulf, A, D. Constales, C. Stal, and T. Nuttens. 2012. Accuracy ascpects of processing and filtering of multibeam data: grid modeling versus TIN based modeling. Paper read at FIG Working Week, 6-10 May 2012, at Rome, Italy: 6 p. (on CD-ROM).

Höhle, J., and M. Höhle. 2009. "Accuracy assessment of digital elevation models by means of robust statistical methods." ISPRS International Journal of Photogrammetry and Remote Sensing no. 64 (4):398-406.

Krzystek, P. 2003. "Filtering of Laser Scanning Data in Forest Areas using Finite Elements." International Archives of Photogrammetry and Remote Sensing no. 34 (3):6 p. (on CD-ROM).

Shewchuk, J. 1996. "Triangle: engineering a 2D quality mesh generator and Delaunay triangulator" In Applied Computational Geometry Towards Geometric Engineering, edited by L. Ming and M. Dinesh, 203-222. Heidelberg, Germany: Springer.

Sithole, G. 2001. "Filtering of laser altimetry data using a slope adaptive filter." International Archives of Photogrammetry and Remote Sensing no. 34 (3):203-210.

Vosselman, G. 2000. "Slope based filtering of laser altimetry data." International Archives of Photogrammetry and Remote Sensing no. 33 (B3):935-942.

Wack, R., and A. Wimmer. 2002. "Digital terrain models from airborne laser scanner data: a grid based approach." International Archives of Photogrammetry and Remote Sensing no. 33 (Part 3):293-296.

Zhang, K., S. C. Chen, D. Whitman, M. L. Shyu, J. Yan, and C. Zhang. 2003. "A progressive morphological filter for removing nonground measurements from airborne LiDAR data." IEEE Transactions on Geoscience and Remote Sensing no. 41 (4):872-882. 